\title{
Conceptualización y taxonomía para estructurar los conocimientos acerca de la ciencia
}

\author{
María-Antonia Manassero-Mas \\ Departamento de Psicología, Universidad de las Islas Baleares, Palma de Mallorca, España. \\ ma.manassero@uib.es \\ ORCID: http:/ / orcid.org/0000-0002-7804-7779 \\ Ángel Vázquez-Alonso \\ Centro de Estudios de Posgrado, Universidad de las Islas Baleares, Palma de Mallorca, España. \\ angel.vazquez@uib.es \\ ORCID: http:/ / orcid.org/0000-0001-5830-7062
}

[Recibido: 11 Diciembre 2018. Revisado: 4 Mayo 2019. Aceptado:19 Junio 2019]

\begin{abstract}
Resumen: Los investigadores en didáctica de la ciencia coinciden en que los meta-conocimientos acerca de la ciencia o naturaleza de la ciencia $(\mathrm{NdC})$ son un componente constitutivo y relevante de la alfabetización científica para todos. Este acuerdo básico contrasta con la importante controversia acerca de los contenidos más apropiados para representar $\mathrm{NdC}$ en el currículo escolar, entre la "visión de consenso", (así denominada en la literatura), y sus críticos, que la consideran un reduccionismo empobrecedor, y proponen conceptualizaciones más amplias. Partiendo del modelo filosófico de los tres mundos sobre la ciencia, este estudio elabora una conceptualización sobre los meta-conocimientos interdisciplinarios de $\mathrm{NdC}$, epistémicos y no epistémicos, que permite generar y justificar esos conocimientos de forma natural desde el modelo conceptual, e incorpora la visión de consenso y sus críticas. Desde este marco, se propone una taxonomía flexible y abierta, cuya justificación surge de su funcionalidad para englobar las categorías emergentes del modelo conceptual, así como las diversas propuestas anteriores de distintos autores. La taxonomía está formada por cuatro aspectos básicos, cada uno con múltiples temas y subtemas específicos, articulados de forma flexible y abierta para admitir nuevas extensiones y facilitar a los no especialistas, especialmente el profesorado de ciencias, una fácil comprensión de los contenidos meta-cognitivos del currículo.
\end{abstract}

Palabras clave: Alfabetización en ciencia y tecnología; ciencia, tecnología, sociedad, ambiente; Conceptualización de la naturaleza de la ciencia y la tecnología; enseñanza y aprendizaje.

\section{Conceptualization and taxonomy to structure the knowledge about science}

Abstract: Science education researchers agree that meta-knowledge about science or nature of science (NoS) is a constitutive and relevant component of scientific literacy for all. This basic agreement contrasts with the important controversy about the most appropriate contents to represent $\mathrm{NoS}$ in the school curriculum, between the "consensus view" (so called in the literature), and its critics, who consider it an impoverishing reductionism, and propose broader conceptualizations. Starting from the three-world philosophical model about science, this study elaborates a conceptualization that allows to generate and justify the epistemic and non-epistemic interdisciplinary meta-knowledge about science from the conceptual model, assuming the consensus view and its criticisms. From this framework, a flexible and open taxonomy is proposed, whose justification arises from its functionality to encompass the emergent categories of the conceptual model, as well as the previous proposals from different authors. The taxonomy is organized through four basic aspects, each displaying multiple specific themes and sub-themes, articulated in a flexible and open way to admit new extensions and to facilitate the nonspecialists, especially science teachers, easy understanding of the curriculum meta-cognitive contents.

Keywords: Scientific and technological literacy; science, technology, society, environment; conceptualization of the nature of science and technology; teaching and learning.

Para citar este artículo: Manassero-Mas, M.A. y Vázquez-Alonso, A. (2019) Conceptualización y taxonomía para estructurar los conocimientos acerca de la ciencia. Revista Eureka sobre Enseñanza y Divulgación de las Ciencias 16(3), 3104. doi: 10.25267/Rev_Eureka_ensen_divulg_cienc.2019.v16.i3.3104 


\section{Introducción}

Este estudio presenta una reflexión sobre la conceptualización de los meta-conocimientos acerca de la ciencia, también denominados ciencia, tecnología y sociedad o naturaleza de la ciencia $(\mathrm{NdC})$, como componente esencial de la alfabetización científica y tecnológica funcional, al servicio de los ciudadanos, y, por lo tanto, desde la perspectiva de la enseñanza y el aprendizaje de las cuestiones que conforman este amplio campo de investigación didáctica y educativa. Esta perspectiva educativa y alfabetizadora para estudiantes actuales surge sobre todo de lo que este campo es hoy, y no tanto de su pasado, que sería una perspectiva más erudita para especialistas (sociólogos, filósofos e historiadores). Se propone un marco teórico actualizado, amplio y genuino para $\mathrm{NdC}$, y, como complemento, se elabora una taxonomía sistémica que guíe la investigación, la enseñanza y el aprendizaje (Allchin, Andersen y Nielsen 2014).

Desde esta perspectiva educativa y actual, la propuesta introduce la tecnología como un nuevo factor ineludible para comprender adecuadamente cómo es la ciencia actual, pues, en los últimos años y en muchas áreas de la investigación (aunque no en todas), existe un proceso de integración entre ciencia y tecnología, que difumina su distinción, y ha propuesto el constructo tecno-ciencia (Tala 2009). Esta integración no pretende sostener una identidad óntica entre ciencia y tecnología, sino solo tener en cuenta el hecho que la tecnología se viene haciendo más científica, y al mismo tiempo, la práctica científica depende cada vez más de la tecnología (Acevedo 2006). La incorporación de las relaciones ciencia-tecnología a los temas de NdC se materializa aquí ampliando la etiqueta a naturaleza de ciencia y tecnología (en lo sucesivo, $\mathrm{NdCy}$ ). Por otro lado, esta integración permite abordar de forma natural la práctica científica y de ingeniería en la educación, tal como se presentan integradas en planes de estudios recientes para las nuevas generaciones (NGSS 2013) y en el acrónimo STEM.

La cuestión nominalista acerca del mejor nombre para designar estos tópicos (tales como $\mathrm{NdC}, \mathrm{NdCy}$ T, CTS, ciencia total, rasgos o ideas de ciencia, etc.) que enseñan a los estudiantes cómo funciona la ciencia, fue zanjada por Aikenhead (2003) con una posición ecléctica donde se relativizaba la cuestión del nombre. Vesterinen, Manassero y Vázquez (2014) delinean la evolución de nombres y tendencias en las últimas décadas, y en particular, las relaciones entre el movimiento CTS y NdC, coincidiendo con Acevedo y García (2016). Lo esencial: NdC engloba un conjunto de conocimientos interdisciplinares y meta-teóricos acerca de la ciencia, epistémicos y no epistémicos, cuya funcionalidad es representar una imagen auténtica y global de la ciencia con la tecnología y la sociedad.

Este estudio elabora una conceptualización (denominada 4-mundos) que es alternativa a la "visión de consenso" (así llamada en la literatura), pues asume las críticas generales planteadas sobre esa visión. También es alternativa a la re-conceptualización de Erduran y Dagher (2014), (RFN a partir de aquî), pues parte de un marco filosófico diferente, que permite generar un modelo y proponer una taxonomía, más amplios y flexibles, que integran múltiples y complejas características de NdC. Algunas ideas germinales del modelo y la elaboración y aplicación de la taxonomía han sido presentadas en otros lugares (Vázquez y Manassero 2015; 2017; Vázquez, Manassero y Bennássar 2015) y en este estudio se elabora la propuesta en profundidad e integrando modelo y taxonomía.

\section{Contextualización teórica}

La naturaleza de la ciencia y tecnología $(\mathrm{NdCy} T)$ se refiere al conjunto de meta-conocimientos elaborados desde múltiples disciplinas (historia, filosofía, y sociología - como meta-ciencias clásicas - y otras, como psicología, economía, ética, etc.) sobre los aspectos ontológicos, 
epistemológicos y sociales acerca de los conocimientos y prácticas científicas y tecnológicas (Lederman 2007, Matthews 2012). Esta interdisciplinariedad configura NdCyT como conocimientos meta-teóricos (conocimiento sobre conocimiento), complejos (interdisciplinar), dialécticos (evolutivo), multifacéticos (perspectivas analíticas múltiples), y controvertidos (argumentativos) que conforman un territorio de investigación permanentemente impugnado. Por ello, su enseñanza y aprendizaje es un desafío, difícil e innovador para los docentes (Millar 2006). La conceptualización propuesta pretende contribuir a que investigadores, didactas y, sobre todo, docentes, puedan comprender mejor este complejo campo.

\section{Alfabetización científica y tecnológica}

Los académicos proponen dos componentes principales para la alfabetización científica ( $\mathrm{y}$ tecnológica) (ACT) para todos los ciudadanos (no solo para científicos o ingenieros): la comprensión "de" la ciencia (conceptos, leyes, modelos y teorías y procesos) y la comprensión "acerca de" la ciencia $(\mathrm{NdCy} \mathrm{T})$. Por lo tanto, enseñar y aprender $\mathrm{NdCy}$ T es un componente central de la alfabetización, y ello fundamenta su enseñanza (Hodson 2008).

En la literatura, la enseñanza de $\mathrm{NdCyT}$ ha sido justificada por razones socio-económicas, culturales, emancipadoras, utilitarias, democráticas y éticas (Sjøberg 1997). Algunas pedagogías tradicionales de enseñanza (por ejemplo, la memorización), al estar vacías de $\mathrm{NdCy} \mathrm{T}$, causan un aprendizaje sin sentido que choca a menudo con los valores constitutivos y la funcionalidad de la ciencia (por ejemplo, sembrando credulidad en lugar de escepticismo crítico) que la NdCyT defiende (Aikenhead 2006).

Esta situación no es satisfactoria para los intereses, necesidades o autoimagen de los estudiantes, que se sienten alienados y desafectos; por ello, muchas mujeres se sienten incómodas cuando aprenden ciencias y muchos estudiantes rechazan la ciencia y la tecnología para sus futuras carreras y trabajos. En cambio, la presencia activa de NdCy'T en las aulas puede proporcionar un sentido de coherencia global a la educación científica, porque la enseñanza de $\mathrm{NdCy}$ T es una fuente de valores que contribuye a una alfabetización auténtica (Duschl, Maeng y Sezen 2011, Erduran y Dagher 2014). En suma, una razón educativa adicional y poderosa para enseñar $\mathrm{NdCy}$ T surge de su capacidad para dar sentido y significado global a toda la enseñanza y a los aprendizajes científicos para todos, pues todos los elementos educativos deben ser coherentes con las características emanadas de $\mathrm{NdCy} T$ (Bennássar, Vázquez, Manassero y García-Carmona 2010).

$\mathrm{El}$ aprendizaje de NdCyT a menudo desarrolla la alfabetización funcional, que puede servir a los ciudadanos para participar en la ciencia o para tomar decisiones informadas en su vida diaria, pues implica comprender algunas características clave de la práctica en ciencia y tecnología, como el desarrollo y validación del conocimiento: suposiciones, recopilación e interpretación de datos, fiabilidad de conclusiones y relaciones entre ciencia, tecnología y sociedad (financiación, comunicación, instituciones, políticas públicas, etc.), y donde los problemas ambientales y socio-científicos son centros de interés prominentes (Allchin et al. 2014, Bybee 1997).

\section{La visión de consenso}

La literatura ha venido en denominar visión de consenso a un enfoque de $\mathrm{NdC}$ concretado en una lista de aspectos $\mathrm{NdC}$, que se justifica por considerar que una lista corta y clara puede ser suficiente para los objetivos de alfabetizar y para facilitar al profesorado una enseñanza tan compleja. Un representante conspicuo de esta visión serían los siete rasgos del conocimiento científico de Lederman: empírico, cargado de teoría, inferencial, creativo, tentativo, cambiante y socialmente impregnado (Lederman 2007). McComas (2008) desarrolló una lista de principios semejante, pero más larga (cuarta columna de la tabla del anexo). 
Esta visión ha sido criticada por ser excesivamente reduccionista, y, por tanto, inválida para representar adecuadamente la $\mathrm{NdCy}$. Otras impugnaciones son la confusión entre las características ontológicas, filosóficas, sociológicas y éticas de la ciencia, la representación inexacta de la heterogeneidad de la práctica científica, la distorsión del desarrollo histórico de la ciencia y la ignorancia o devaluación de algunas características, como el papel de la tecnología, y los aspectos sociales, verbales y comunicativos en la construcción del conocimiento (Allchin 2011, Duschl y Grandy 2008, Matthews 2012, van Dijk 2012). Por lo tanto, los críticos abogan por una visión más integral e inclusiva de $\mathrm{NdCyT}$ (por ejemplo, Allchin llama "ciencia total"), donde todas las características sobre la ciencia (prácticas cognitivas, epistémicas y sociales, así como los contextos materiales y tecnológicos) sean contenidos válidos para enseñar "acerca de" la ciencia.

\section{Alternativas críticas a la visión de consenso}

A través de un estudio Delphi sobre las opiniones de los docentes, Osborne, Collins, Ratcliffe, Millar y Duschl (2003) desarrollaron nuevas y más amplias categorías para NdC, aportando además fundamento empírico. Propusieron las siguientes categorías: ciencia y tecnología, integración cultural, desarrollo cooperativo del conocimiento, creatividad, análisis e interpretación de datos, método científico, pruebas críticas, certeza, diversidad del pensamiento científico, desarrollo histórico y cuestionamiento, hipótesis y predicción.

Matthews (2012) sugirió cambiar la denominación de NdC a características de la ciencia, para las que propuso un conjunto que dan una imagen más integral y amplia de la ciencia (ver detalles en el anexo, tercera columna). Sostiene que ampliar el campo no significa establecer objetivos educativos de alto nivel, y argumenta explícitamente a favor de objetivos modestos, y que la didáctica debe ser adecuada para permitir la elaboración, discusión e indagación de los estudiantes sobre las cuestiones, en lugar de limitarse a trasmitir y evaluar. Aunque esta propuesta sigue centrada en los aspectos epistémicos, aporta una apertura hacia los procesos, las instituciones y los contextos culturales y sociales donde se produce el conocimiento.

Un análisis empírico sobre centenares de frases acerca de $\mathrm{NdCy} T$, con un exigente criterio de acuerdo, elaboró un amplio catálogo con decenas de frases que alcanzaron acuerdo de expertos como ideas informadas y como ideas ingenuas; esto demuestra que el consenso sobre NdCyT puede abarcar un conjunto muy amplio de enunciados, mucho mayor que una pequeña lista de consenso, corta y reduccionista (Acevedo, Vázquez, Manassero y Acevedo 2007).

En resumen, los críticos coinciden en que la visión de consenso es una representación muy reducida de $\mathrm{NdC}$, y abogan por una visión más holística e inclusiva de $\mathrm{NdC}$ : todas las características relacionadas con hacer ciencia son candidatas para ser enseñadas. Esta visión más amplia de ciencia auténtica (total) no solo incluye principios adecuados, sino que amplía la imagen para incluir las concepciones ingenuas, que también deben ser consideradas, y especialmente en la enseñanza. Además, esta controversia demuestra que cualquier visión de $\mathrm{NdC}$ es parcial y fragmentaria; por lo tanto, una estructuración del campo es necesaria no solo para superar el fragmentarismo, sino también para ayudar a investigadores y profesorado a situarse e identificar adecuadamente sus objetivos en medio de la complejidad del campo.

\section{La reconceptualización de Erduran y Dagher (RFN)}

Erduran y Dagher (2014) elaboran una visión más amplia de NdC, partiendo del modelo filosófico del parecido familiar de Irzik y Nola (2014), para desarrollar la idea de que las diferentes ciencias, como los miembros de una familia, se asemejan y diferencian entre sí en algunos aspectos. Esta orientación trata de explicar y conciliar los aspectos generales de la ciencia y los aspectos específicos de las distintas ramas científicas. 
Esta reconceptualización (en lo sucesivo, modelo RFN, como lo denominan sus autoras) propone dos dimensiones interactivas (cognitivo-epistémica y social-institucional) que abarcan muchos otros aspectos. La dimensión cognitivo-epistémica incluye cuatro categorías: prácticas científicas, objetivos y valores, métodos y reglas metodológicas, y conocimiento científico. La dimensión social e institucional contiene las categorías actividades profesionales, ética científica, certificación social, valores sociales y aspectos organizativos, políticos y financieros de la ciencia, aunque en otras descripciones sucesivas solo aparecen cuatro.

El RFN aporta una visión holística, de acuerdo con una visión integral del conocimiento y la práctica científica y otras categorías familiares, y donde los aspectos específicos que se asignan a las categorías (clasificación, observación, experimentación, cuestiones epistemológicas relevantes para la educación científica, similitudes disciplinarias y especificidad de dominio, etc.) explican las variaciones entre las distintas disciplinas de ciencia y tecnología. El ojo de la ciencia y los anillos heurísticos son dos instrumentos del modelo que organizan los elementos de RFN (tabla 1).

Este estudio presenta una alternativa a la propuesta de RFN, compartiendo las críticas a las listas reduccionistas de principios y la misma concepción global para $\mathrm{NdCy} T$, aunque trata de mejorar algunos aspectos organizativos del campo.

\section{La conceptualización 4-mundos}

La inspiración de partida para esta nueva propuesta es el modelo filosófico de los tres mundos ontológicos para describir la ciencia (Popper 1974), refinado con las aportaciones de Hodson (2008). En ese modelo, el Mundo 1 es el mundo físico de los objetos materiales (tanto naturales como artificiales), mientras que el Mundo 2 se refiere al reino psicológico de los pensamientos, cogniciones, ideas y estados mentales sobre el Mundo 1, desarrollados por las personas (y los científicos), a través de la conciencia, las actividades mentales y las percepciones, desarrolladas por medio de la observación, clasificación y experimentación, que producen datos y pruebas, generadores de variadas ideas sobre el mundo físico (Figura 1).

La elaboración avanzada de las cogniciones (Mundo 2) mediante herramientas personales y sociales, epistémicas y no epistémicas, (comunicación, razonamiento, argumentación, contradicción, replicación, autocrítica, revisión por pares, etc.) crea productos nuevos sobre el Mundo 1, tangibles e independientes de la persona (leyes, modelos, teorías, ilustraciones, artesanías, problemas, argumentos, libros, documentos, artefactos, obras de arte, etc.) que conforman el Mundo 3, denominado por Popper el mundo del conocimiento creado (objetivo). Además, el Mundo 3 y el Mundo 1 retroalimentan continuamente al Mundo 2 con prácticas innovadoras y originales (a menudo, nuevas observaciones y experimentos inspirados en las nuevas teorías), de modo que el conocimiento científico y tecnológico validado (Mundo 3) puede detectar contradicciones y sugerir orientaciones innovadoras para prácticas o replicaciones nuevas (carga teórica de las observaciones). A su vez, los nuevos datos, pruebas y resultados extraídos del Mundo 1 (anomalías, resultados cruciales, etc.) pueden influir en las nuevas prácticas en el Mundo 2 (evoluciones o revoluciones) y producir desarrollos y refinamientos nuevos del conocimiento (Mundo 3), a través de estos procesos continuos e interactivos. 


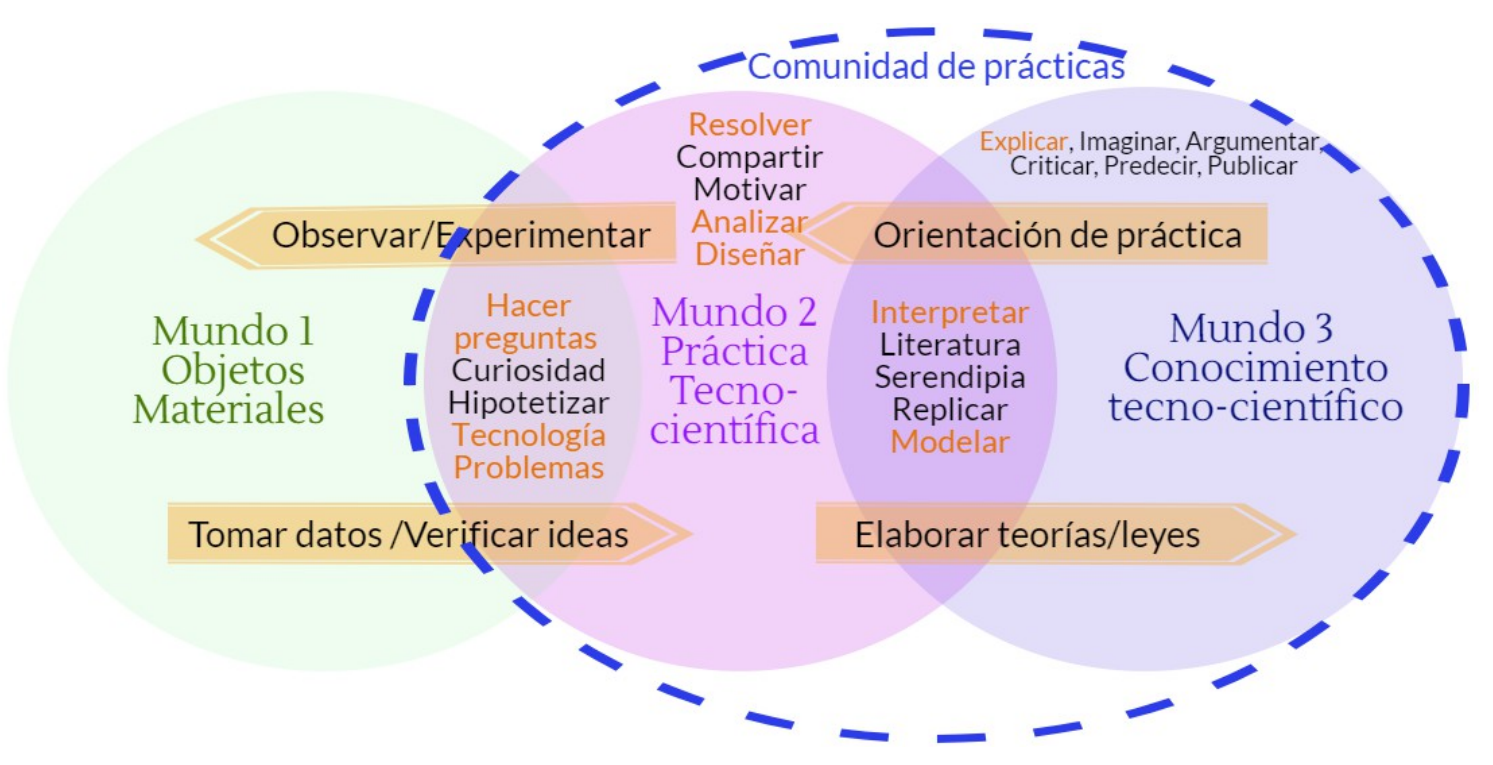

Figura 1. El modelo de los tres mundos de Popper sobre la ciencia, revisado por Hodson, aplicado a la reconceptualización de los temas de la naturaleza de la ciencia y la tecnología

Además de los estados mentales de los científicos, el Mundo 2 desarrolla una amplia gama de complejas interacciones entre los científicos (controversias y debates científicos) y entre la comunidad científica y la sociedad en general (impactos sociales). Por otro lado, las interacciones intensas, permanentes y dialécticas entre el Mundo 2 y el Mundo 3 imbrican ambos mundos tan profundamente, que la distinción entre conocimiento objetivo (Mundo 3) y subjetivo (Mundo 2) se vuelve difusa. De hecho, Hodson (2008) descartó la objetividad como propiedad del Mundo 3, llamando al Mundo 3 "mundo del conocimiento científico y tecnológico" (teorías y artefactos científicos y tecnológicos) y al Mundo 2 "mundo de la práctica científica" (pensamientos científicos subjetivos y acciones sobre M1 y M3). También adiciona la tecnología al modelo de ciencia (de acuerdo con la propuesta de la introducción).

Además, Hodson amplía la complejidad del M2 a todo tipo de actividades humanas, personales y sociales, reconociendo la influencia de colegas, gobiernos, empresas, y por supuesto, las instituciones educativas, e incluso los fraudes y las actividades pseudo-científicas y a todas las personas relacionadas con ciencia y tecnología (científicos, tecnólogos, profesores, estudiantes y ciudadanos).

El modelo adaptado de los 3-mundos permite su aplicación conceptual para defender o discutir cualquier aspecto propio de la práctica científica, aunque aquí se aplica para conceptualizar, exacta y holísticamente, la práctica y el conocimiento científico. Por ejemplo, la equilibrada representación gráfica de los tres mundos de la figura 1 podría alterarse (haciendo M3 más grande y M2 minúsculo), para representar la educación tradicional de la ciencia, caracterizada por la preponderancia de M1 y M3 (hechos y conocimientos) y exclusión de la enseñanza de M2 (práctica) o conocimientos acerca de la ciencia (figura 2). Por el contrario, una representación con M2 de mayor tamaño (como la figura 1) representaría una educación más centrada en la práctica científica (sin olvidar los conocimientos). Este modelo permite a profesores y estudiantes entender y asumir muy intuitivamente la idea de que, en la educación científica escolar, reflexionar acerca de la ciencia importa tanto como conocer la ciencia, y, tal vez, sea más útil. 


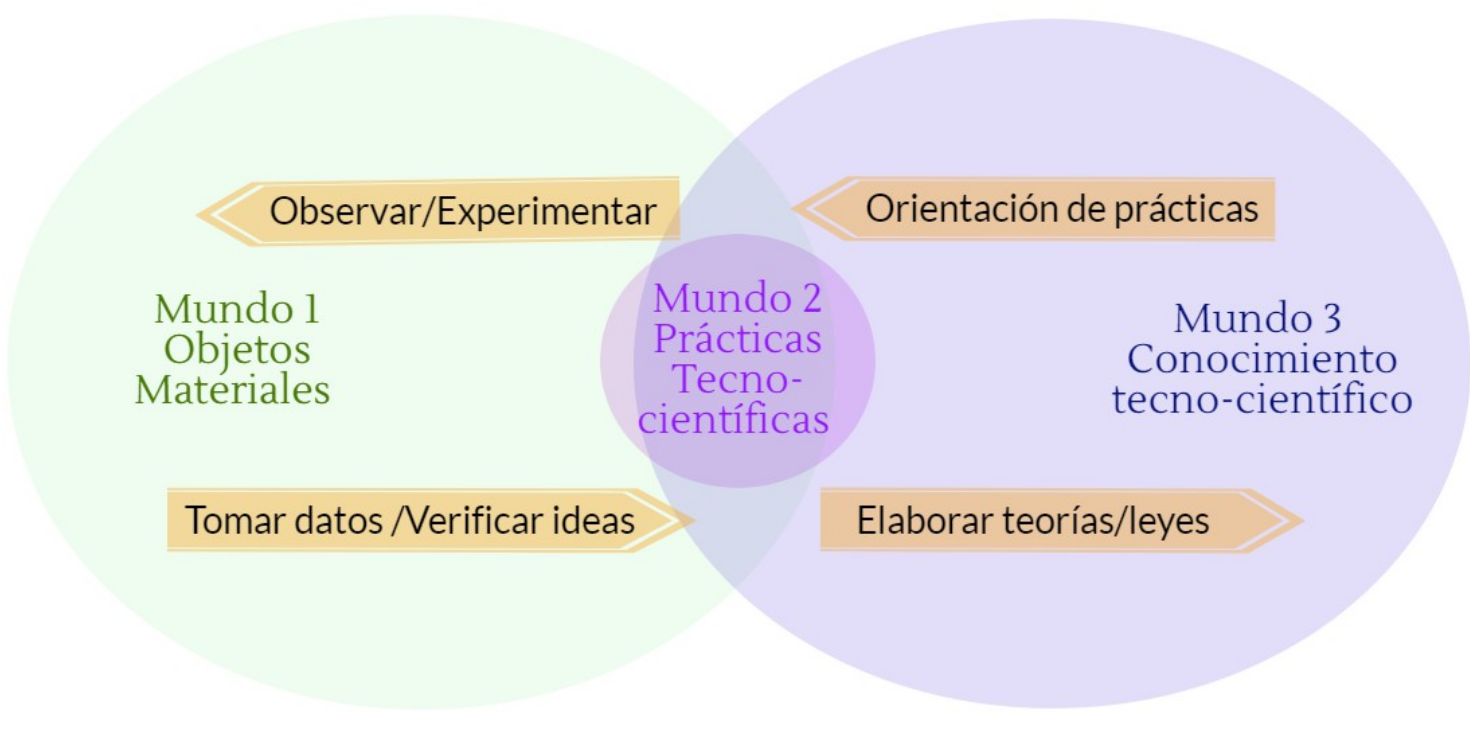

Figura 2. El modelo de los tres mundos de Popper sobre la ciencia, revisado por Hodson, aplicado a representar la educación tradicional de la ciencia. Se visualiza el dominio del conocimiento de hechos (M1) y teorías científicas (M3) y se excluyen los temas de la naturaleza de la ciencia y la tecnología (M2); también sugiere una epistemología muy positivista, mediante el enlace directo entre naturaleza (M1) y conocimiento (M3).

La imbricación y complejidad de relaciones e interacciones entre el Mundo 2 y el Mundo 3 conforma una nueva entidad (comunidad de prácticas) que combina conocimiento y estados mentales, actores individuales y colectivos, en un sistema complejo formado por una densa red de relaciones e interacciones mutuas entre la sociedad, los actores y el conocimiento, que ha sido fundamentalmente estudiada desde la sociología del conocimiento científico (Bazzul y Bencze 2010). Algunas de actividades frecuentes en esta comunidad de prácticas se expresan en forma de verbos de acción (figura 1): hacer preguntas, resolver, diseñar, interpretar, explicar, imaginar, argumentar, predecir, publicar, etc. La comunidad de prácticas es el escenario donde se promueve la crítica seria, propia del escepticismo científico, entre todas las personas implicadas en el sistema (por ejemplo, discutir los enfoques realistas o instrumentalistas de la ciencia); en un contexto educativo, este escenario ofrece una guía para trasladar el pensamiento (meta)científico al aprendizaje.

De las intensas interacciones y realimentaciones internas sociales y organizativas entre científicos, y entre la comunidad tecno-científica y la sociedad global, propias de los mundos M1-M2-M3, surge una espiral interminable de innovaciones y desarrollos. Cuando desde diferentes disciplinas sociales (filosofía, sociología, historia, psicología, política, economía, etc.) se estudian el conocimiento (Mundo 3), las actividades en el Mundo 2, y los impactos sobre el Mundo 1 y la sociedad de las interacciones dentro de la comunidad de prácticas, se genera un nuevo meta-conocimiento sobre CyT (sobre el conocimiento, los estados mentales y los actores de la Cy'T en la sociedad), que constituye un nuevo Mundo 4 (meta-conocimientos de $\mathrm{Cy} \mathrm{T}$ ), que crea una nueva forma de conocimiento interdisciplinar, complejo, multifacético, crítico, dialéctico y controvertido sobre el sistema de ciencia y tecnología, que es continuamente informado, influenciado y transformado por las actividades contingentes de la comunidad de prácticas (Vázquez y Manassero 2017). 


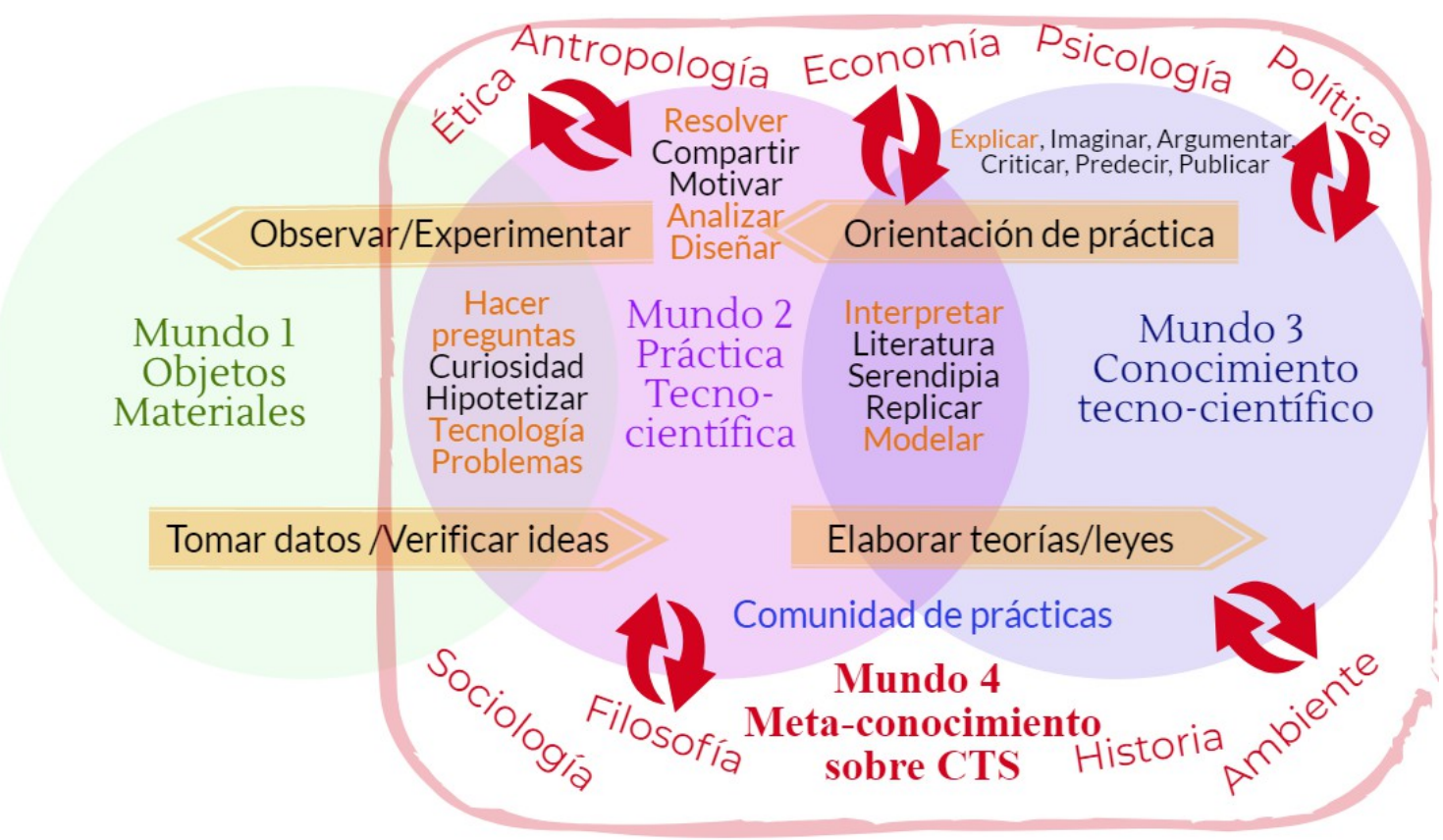

Figura 3. Modelo meta-teórico para la re-conceptualización del campo NdCyT, basada en los tres mundos de Popper; el núcleo es un meta-conocimiento interdisciplinar (Mundo 4) que surge del estudio de la comunidad de prácticas científicas desde diferentes disciplinas (principalmente sociología, filosofía, historia, y otras como psicología, economía, etc.).

La educación se puede considerar un subsistema dentro de la comunidad de prácticas y el modelo del Mundo 4 sugiere una propuesta educativa innovadora: la inclusión de sus metaconocimientos acerca de CýT como contenido del currículo escolar. Estos contenidos incluyen impactos, comunicación, validación, información y análisis del conocimiento científico (ver detalles en la tabla del anexo). Esta primera elaboración de la propuesta teórica de re-conceptualización de la $\mathrm{NdCyT}$ en el modelo expuesto (denominado en lo sucesivo 4mundos) da cuenta también de los conocimientos básicos sobre $\mathrm{NdCy} T$ a ser enseñados.

Resumiendo, la conceptualización 4-mundos desarrolla las interacciones mutuas entre los tres mundos para mostrar y explicar la génesis de los contenidos complejos, multifacéticos y controvertidos y las estructuras que presentan y caracterizan ciencia y tecnología en el mundo actual. En particular, el meta-conocimiento interdisciplinario del Mundo 4 creado a partir del estudio de contenidos y estructuras de los Mundos 1, 2 y 3 constituye el núcleo de contenidos de NdCyT para la educación científica (Vázquez y Manassero 2017).

\section{Un marco taxonómico para la conceptualización de $\mathrm{NdCy} T$}

Toda la investigación sobre $\mathrm{NdCy}$, desde las listas de la visión de consenso hasta el modelo de Erduran y Daher, tienen el objetivo implícito de organizar la complejidad del campo, como guía para la investigación y la educación. El modelo 4-mundos ilustra la generación de los complejos meta-conocimientos de $\mathrm{NdCy}$, cuya gran riqueza exige un sistema de clasificación que los organice para manejarlos con eficacia. Aunque cualquier clasificación de la realidad tiene siempre aspectos de arbitrariedad y convenio, su justificación resultará en gran medida de su eficacia para cumplir bien su función de representación y guía en este campo.

Para el modelo 4-mundos se utiliza un marco taxonómico elaborado por los autores, a partir de la propuesta de Aikenhead y Ryan (1992) y, cuya justificación surge de la investigación que ya lo ha aplicado (Bennássar et al. 2010) y de su mayor funcionalidad para representar el campo 
(como se mostrará más abajo). Esta taxonomía propone como organizadores cuatro aspectos básicos:

I. Definiciones e interacciones entre ciencia y tecnología.

II. Sociología externa de la ciencia y la tecnología

III. Sociología interna de la ciencia y la tecnología

IV. Epistemología.

La naturaleza de los aspectos I y IV es epistémica y cognitiva, mientras que los aspectos II y III reúnen las múltiples facetas de las relaciones sociales e institucionales de CyT; cada uno de estos cuatro aspectos básicos, a su vez, se desarrollan en varios temas (tabla 1). Después, cada tema se divide de manera abierta y flexible en varios subtemas (ver la primera columna del anexo), que ayudan a la taxonomía a cumplir con precisión su función de proporcionar una clasificación sistemática, flexible, abierta y concreta que permite incluir todos los potenciales contenidos y problemas acerca de $\mathrm{NdCy}$.

Tabla 1. Comparación y equivalencia entre los aspectos y temas del modelo y de la taxonomía 4-mundos y las dimensiones y categorías del modelo RFN de Erduran y Dagher (2014).

\begin{tabular}{|c|c|c|c|}
\hline \multicolumn{2}{|c|}{ Modelo 4-mundos (taxonomía VOSTS) } & \multicolumn{2}{|c|}{ Modelo RFN } \\
\hline Aspectos & Temas & Dimensiones & Categorías \\
\hline \multirow{2}{*}{ I - DEFINICIONES } & \multirow{2}{*}{ 1. Ciencia y tecnología } & \multirow{4}{*}{$\begin{array}{l}\text { SISTEMA } \\
\text { EPISTÉMICO Y } \\
\text { COGNITIVO }\end{array}$} & Objetivos y valores \\
\hline & & & Prácticas científicas \\
\hline \multirow{2}{*}{$\begin{array}{l}\text { IV - } \\
\text { EPISTEMOLOGÍA }\end{array}$} & \multirow{2}{*}{$\begin{array}{l}\text { 9. Naturaleza del } \\
\text { conocimiento científico }\end{array}$} & & Reglas metodológicas \\
\hline & & & $\begin{array}{l}\text { Conocimiento científico } \\
\text { (teorías, leyes y modelos) }\end{array}$ \\
\hline \multirow{5}{*}{$\begin{array}{l}\text { II - SOCIOLOGÍA } \\
\text { EXTERNA }\end{array}$} & $\begin{array}{l}\text { 2. Influencias de la socie- } \\
\text { dad en C\&T }\end{array}$ & \multirow{8}{*}{$\begin{array}{l}\text { SISTEMA SOCIAL- } \\
\text { INSTITUCIONAL }\end{array}$} & \\
\hline & $\begin{array}{l}\text { 3. Influencias } \\
\text { tridimensionales C-T-S }\end{array}$ & & \\
\hline & $\begin{array}{l}\text { 4. Influencias de C\&T en la } \\
\text { sociedad }\end{array}$ & & $\begin{array}{l}\text { Organizacionales, } \\
\text { políticas y financieras }\end{array}$ \\
\hline & \multirow{2}{*}{$\begin{array}{l}\text { 5. Influencias de la ciencia } \\
\text { escolar en la sociedad }\end{array}$} & & Valores sociales \\
\hline & & & $\begin{array}{l}\text { Certificación y } \\
\text { divulgación social }\end{array}$ \\
\hline \multirow{3}{*}{$\begin{array}{l}\text { III - SOCIOLOGÍA } \\
\text { INTERNA }\end{array}$} & $\begin{array}{l}\text { 6. Características de los } \\
\text { científicos }\end{array}$ & & Ética científica \\
\hline & $\begin{array}{l}\text { 7. Construcción social del } \\
\text { conocimiento científico }\end{array}$ & & Actividades profesionales \\
\hline & $\begin{array}{l}\text { 8. Construcción social de la } \\
\text { tecnología }\end{array}$ & & \\
\hline
\end{tabular}

Un primer dato que justifica la validez de la taxonomía VOSTS del modelo 4-mundos son las coincidencias y diferencias con la clasificación del modelo RFN. La principal coincidencia es la imagen global para la conceptualización de $\mathrm{NdC}$ que proporcionan ambas propuestas. La tabla 1 evidencia que ambos sistemas clasifican los rasgos de $\mathrm{NdC}$ mediante constructos amplios, que pueden considerarse equivalentes. RFN presenta dos dimensiones (epistémica-cognitiva y social-institucional), cuya correspondencia con el modelo 4-mundos es bastante clara: los aspectos definiciones y epistemología se corresponderían con la dimensión cognitiva del modelo RFN, y los aspectos sociología externa e interna se corresponderían con la dimensión 
social-institucional del modelo RFN. Las dimensiones y categorías de RFN son un caso especial del modelo 4-mundos, es decir, que nuestro modelo incluye como caso particular el modelo RFN.

Las diferencias surgen de los defectos detectados en el análisis del desarrollo de algunas categorías en el modelo RFN. Por ejemplo, la dimensión epistémica y cognitiva del sistema RFN parece más desarrollada que la dimensión social e institucional, y especialmente, la categoría de prácticas científicas. Este hiper-desarrollo lleva a cuestionar la clasificación de algunos temas asignados a esta dimensión, puesto que tal vez podrían clasificarse mejor como sociales en lugar de epistémicos. Este podría ser el caso de algunas categorías, tales como las argumentaciones críticas, las evaluaciones y las reflexiones sobre la práctica, y el análisis de las desventajas y riesgos de los desarrollos científicos y tecnológicos. Observaciones semejantes también pueden extenderse a la dimensión institucional, por ejemplo, los asuntos organizativos, políticos y financieros de la ciencia, no están completamente desarrollados en la propuesta RFN. Por el contrario, los muchos subtemas del sistema 4-mundos, especialmente para los temas sociales (véase la primera columna del anexo) pueden ejemplificar y compensar la falta de desarrollo de muchas categorías sociales del modelo RFN (consultar la primera y la segunda columna del anexo).

Otras diferencias entre los modelos RFN y 4-mundos se refieren al papel y a la consideración de la tecnología, como un compañero importante de la ciencia actual, y una referencia crucial a los muchos aspectos de la sociología externa de la ciencia (desde la ambiental a la energía y el bienestar social). Mientras que el modelo RFN ofrece una explicación genérica de las relaciones ciencia-tecnología-sociedad, el modelo 4-mundos proporciona una consideración más detallada y explícita de estas relaciones y del papel de la tecnología.

Por otro lado, el sistema RFN presenta diferentes etiquetas para categorías aparentemente similares, como es el caso de los valores y las prácticas. Así, los valores aparecen bajo las siguientes etiquetas: objetivos y valores, valores sociales y ética científica; las prácticas aparecen en la forma de prácticas científicas y prácticas profesionales. Esta multiplicidad de etiquetas parece excesiva cuando, aparentemente, se refieren a la misma entidad; además, esta innecesaria multiplicidad puede poner en riesgo la descripción integral de $\mathrm{NdC}$ y llevar a una cierta confusión conceptual del concepto representado en cada caso.

El modelo 4-mundos proporciona una poderosa representación para conceptualizar $\mathrm{NdCyT}$ para la educación, ya que las dimensiones y categorías de $\mathrm{NdCy} T$ surgen de forma natural de las interacciones y relaciones entre los tres mundos (figura 3). Además, la clasificación de las características de $\mathrm{NdCy}$ T en la taxonomía 4-mundos es amplia y lo suficientemente flexible como para incluir fácilmente cualquier problema nuevo que pueda aparecer dentro de este campo. De hecho, todos los temas de $\mathrm{NdC}$ propuestos por los autores citados en este estudio pueden asignarse a alguno de los subtemas de la taxonomía VOSTS, como muestra la tabla más extensa del anexo. Especialmente, todas las categorías presentadas por la conceptualización RFN de Erduran y Dagher (2014) corresponden al menos a un subtema del modelo 4-mundos; sin embargo, muchos subtemas del modelo 4-mundos carecen de correspondencia con el modelo RFN e incluso con otros autores, lo que demuestra que el modelo 4-mundos engloba las propuestas anteriores para temas de $\mathrm{NdC}$.

Además, el contenido y la estructura son suficientemente claros como para ayudar a los no especialistas y al profesorado a ubicar cualquier tema dentro de la taxonomía, planificarlos para que sean enseñados, comprender su importancia para la educación científica y tecnológica y desarrollar una heurística para desplegar las lecciones. En resumen, facilita a los docentes el diseño de planes explícitos para enseñar cualquier tema de $\mathrm{NdCyT}$ a sus estudiantes. 
Finalmente, la taxonomía de la conceptualización 4-mundos ha venido probando en los últimos años su efectividad para investigar y desarrollar currículos y programas educativos, y especialmente para hacer frente a la formación de los docentes de ciencia y tecnología, como un grupo objetivo principal (Bennássar et al. 2010; Vázquez y Manassero 2013; Vázquez, Manassero y Bennássar 2014).

\section{Conclusiones}

Este estudio presenta una conceptualización de NdCyT basada en el modelo 4-mundos y la taxonomía VOSTS que ofrece un marco global enfocado para la educación en ciencia y tecnología, cuya visión global es alternativa a la visión de consenso, basada en listas simples. Sin embargo, comparte la concepción global de la ciencia y muchos problemas con otros autores, especialmente, con la re-conceptualización RFN de Erduran y Dagher (2014).

Ambas conceptualizaciones tienen un alto grado de convergencia en características clave para el campo, como asumir una visión holística básica sobre la $\mathrm{NdC}$, las críticas a la visión de consenso, un mapa coincidente que desarrolla cuestiones específicas similares y la proyección sobre la educación en ciencia y tecnología. Pero los modelos generadores de ambas conceptualizaciones son diferentes: RFN se basa en la filosofía del parecido familiar y heurísticos, mientras que 4-mundos se basa en la filosofía de los tres mundos de Popper, que integra la naturaleza de la tecnología y la práctica de ingeniería.

El modelo 4-mundos mejora la propuesta RFN porque proporciona una poderosa representación para $\mathrm{NdC}$, donde los aspectos y temas surgen directamente de la elaboración de las interacciones y las relaciones naturales entre los tres mundos, que son lo suficientemente amplias para cubrir todo el campo que conceptualiza, y también son lo suficientemente flexibles como para estar abiertos a nuevos escrutinios y posterior adaptación a las nuevas realidades futuras de la ciencia y la tecnología. Los aspectos y temas de 4-mundos surgen de forma natural del sencillo y potente modelo de interacciones (M2 engendra la dimensión social y M3 engendra la dimensión epistemológica), mientras que la heurística de RFN no permite desarrollar una justificación tan explícita. El análisis de la comunidad de prácticas desde la perspectiva de otras disciplinas externas, y ajenas a la ciencia y la tecnología, da lugar de una manera natural a la dimensión meta-cognitiva que caracteriza el meta-conocimiento de $\mathrm{NdCy}$ T.

La conceptualización 4-mundos muestra un número mayor y más estructurado de aspectos (4), temas (9) y subtemas (más de 50) que las dimensiones RFN (2) y categorías (más de 20), de modo que el modelo de 4-mundos resulta más amplio e incluye los contenidos RFN como un caso particular. Esta configuración diferencial plantea algunas cuestiones específicas; por un lado, RFN apela a una heurística básica, de sentido común, cuyo limitado alcance prospectivo señala algunas flaquezas, como, por ejemplo, un desequilibrio entre las dos dimensiones RFN (epistémica y social), donde el sistema social e institucional parece menos desarrollado que el cognitivo-epistémico.

Además, algunas categorías de la dimensión social e institucional de RFN aparecen confusas. Por ejemplo, RFN atribuye una categoría de objetivos y valores en la ciencia en ambas dimensiones (epistémica y social) de modo que su papel aparece mistificado y controvertido entre ambas dimensiones, y además, la categoría está menos desarrollada dentro del sistema social. Asimismo, la repetición de varias categorías que involucran valores no está muy justificada (ethos científico contra valores sociales), pues la naturaleza compleja y transversal de los valores no está bien desarrollada. RFN presenta algunas categorías (por ejemplo, ética científica, aspectos organizativos, políticos y financieros de la ciencia, crítica científica) que no aparecen en otras partes de la propuesta. Por último, la asignación de algunos aspectos de 
RFN a una categoría específica también es polémica, por su superposición en varias categorías (por ejemplo, la revisión por pares podría estar asignada a la certificación social o a actividades profesionales).

Más allá de las diferencias, se debe enfatizar nuevamente que ambas propuestas comparten una explicación holística para la representación de la ciencia que intenta superar las visiones reduccionistas de la visión de consenso, integrando y ampliando contenidos, métodos (enseñanza, aprendizaje, investigación ...) y la creación de herramientas de desarrollo dinámicas e interactivas.

Desde la perspectiva de la educación en ciencia y tecnología, el modelo 4-mundos aporta una representación flexible, intuitiva y útil que permite dar sentido a la enseñanza como un organizador de ideas acerca de la ciencia. Profesores y estudiantes pueden fácilmente establecer vínculos entre el conocimiento, la práctica y la naturaleza, que inducen a superar la enseñanza tradicional de la ciencia y tecnología centrada preponderantemente en el mundo de los conocimientos (M3), para abrirse a una variedad de combinaciones pedagógicas de métodos, contextos, temas y épocas históricas, que explícitamente permitan una auténtica comprensión total de la ciencia. Al establecer una relación natural entre conocimiento (M3) y el conocimiento acerca de la ciencia $(\mathrm{NdC})$ de $\mathrm{M} 4$ y $\mathrm{M} 2$, el modelo de 4-mundos ayuda a crear un espacio de reflexión de profesores y estudiantes en torno a los aspectos más difíciles, como el cambio de mentalidades y la comprensión de la relación dialéctica y cambiante entre conocimientos, práctica y naturaleza, que es más eficaz que el mero aprendizaje de conocimiento abstracto tradicional (Vázquez y Manassero 2016).

No obstante, los fundamentos teóricos de este nuevo enfoque aún deben continuar desarrollándose para ampliar su validación empírica y las pruebas de su efectividad para el desarrollo curricular y estrategias de enseñanza, a través del trabajo de los responsables políticos, los creadores de currículos, los investigadores, los profesores de ciencias, y otros interesados. Dentro de esta perspectiva, debe tenerse en cuenta que la investigación (especialmente la literatura sobre la enseñanza de cuestiones socio-científicas, la innovación responsable de la investigación y otros temas) está empezando a señalar insistentemente que la falta de habilidades de pensamiento de orden superior (capacidad de pensamiento crítico, de reflexión, de argumentación válida y razonamiento, de toma de decisiones, de solución problemas abiertos ...) son factores cruciales que dificultan la comprensión de los temas de NdCyT. La detallada conceptualización del modelo 4-mundos también puede contribuir a incluir y desarrollar sistemáticamente en educación aquellas habilidades de pensamiento que pueden ser críticas para la enseñanza y el aprendizaje exitosos de los contenidos de $\mathrm{NdCy}$.

\section{Agradecimientos}

Proyecto EDU2015-64642-R (MINECO/FEDER) con financiación del Ministerio de Economía y Competitividad de España y el Fondo Europeo de Desarrollo Regional.

\section{Referencias}

Acevedo J. A. (2006). Modelos de relaciones entre ciencia y tecnología: un análisis social e his tórico. Revista Eureka sobre Enseñanza y Divulgación de las Ciencias 3(2), 198-219.

Acevedo Díaz J.A., García Carmona A. (2016). Algo antiguo, algo nuevo, algo prestado. Tendencias sobre la naturaleza de la ciencia en la educación científica. Revista Eureka sobre Enseñanza y Divulgación de las Ciencias 13(1), 3-19.

Acevedo J. A., Vázquez A., Manassero M. A., Acevedo P. (2007). Consensos sobre la naturaleza de la ciencia: fundamentos de una investigación empírica. Revista Eureka sobre Ensenanza y Divulgación de las Ciencias, 4(1), 42-66. 
Aikenhead G. (2003). STS education: A rose by any other name. En R. Cross (Ed.), A vision for science education: Responding to the work of Peter J. Fensham (pp. 59-75). London: Routledge Press.

Aikenhead G. S. (2006). Science education for everyday life: Evidence-based practice. New York: Teachers College Columbia University.

Aikenhead G. S., Ryan A. G. (1992). The development of a new instrument: "Views on science-technology-society" (VOSTS). Science Education 76(5), 477-491.

Allchin D. (2011). Evaluating knowledge of the nature of (whole) science. Science Education 95(3), 518-542.

Allchin D., Andersen H. M., Nielsen K. (2014). Complementary Approaches to Teaching Nature of Science: Integrating Student Inquiry, Historical Cases, and Contemporary Cases in Classroom Practice. Science Education 98(3), 461-486.

Bazzul J., Bencze J. L. (2010). Adapting Popper's Three Worlds as a tool for thinking about the Nature of Science (NOS). A paper presented at the annual conference of the National Association for Research in Science Teaching, March 20-24, Philadelphia, PA.

Bennássar, A., Vázquez, A., Manassero M. A., \& García-Carmona, A. (Coor.) (2010). Ciencia, tecnología y sociedad en Iberoamérica: Una evaluación de la comprensión de la naturaleza de ciencia y tecnología. Madrid: OEI. Consultado en www.oei.es/salactsi/DOCUMENTO5vf.pdf.

Bybee R. W. (1997). Achieving scientific literacy: From purposes to practices. Portsmouthg: Heinemann.

Duschl R., Grandy R. (Eds.). (2008). Teaching Scientific Inquiry: Recommendations for Research and Implementation. Rotterdam: Sense Publishers.

Duschl R., Maeng S., Sezen A. (2011). Learning progressions and teaching sequences: A review and analysis. Studies in Science Education 47(2), 123-182.

Erduran S., Dagher Z. R. (2014). Reconceptualizing the nature of science for science education, scientific knowledge, practices and other family categories. Dordrecht: Springer.

Hodson D. (2008). Towards scientific literacy: A teachers' guide to the history, philosophy and sociology of science. Rotterdam: Sense Publishers.

Irzik G., Nola R. (2014). New directions for NOS research. En M. Matthews (Ed.), International handbook of research in history, philosophy and science teaching (pp. 999-1022). Dordrecht: Springer.

Lederman N. G. (2007). Nature of science: past, present, and future. En S. K. Abell, N. G. Lederman (Eds.), Handbook of research on science education (pp. 831-879). Mahwah: Lawrence Erlbaum Associates.

Matthews M. R. (2012). Changing the focus: From nature of science (NOS) to features of science (FOS). En M. S. Khine (Ed.), Advances in nature of science research. Concepts and methodologies (pp. 3-26). Dordrecht: Springer.

McComas W. F. (2008). Seeking historical examples to illustrate key aspects of the nature of science. Science \& Education 17, 249-263. 
Millar R. (2006). Twenty first century science: Insights from the design and implementation of a scientific literacy approach in school science. International Journal of Science Education 28(13), 1499-1521.

NGSS Lead States (2013). Next Generation Science Standards: For States, By States. Washington: The National Academies Press.

Osborne J., Collins S., Ratcliffe M., Millar R., Duschl R. (2003). What "ideas-about-science" should be taught in school science? A Delphi study of the expert community. Journal of Research in Science Teacbing 40(7), 692-720.

Popper K. (1974). Conocimiento Objetivo. Madrid: Editorial Tecnos.

Sjøberg S. (1997). Scientific literacy and school science. Arguments and second thoughts. En S. Sjoberg y E. Kallerud (Eds.), Science, technology and citizenship. The public understanding of science and technology in science education and research policy (pp. 9-28). Oslo: NIFU.

Tala S. (2009). Unified View of Science and Technology for Education: Technoscience and Technoscience Education. Science \& Education 18(3-4), 275-298.

van Dijk E. M. (2012). Relevant features of science: Values in conservation biology. Science \& Education 22(9), 2141-2156.

Vázquez, Á., Manassero, M.A. (2013). La comprensión de un aspecto de la naturaleza de ciencia y tecnología: Una experiencia innovadora para profesores en formación inicial. Revista Eureka sobre Enseñanza y Divulgación de las Ciencias, 10, 630-648.

Vázquez, Á., Manassero, M.A. (2015). Una taxonomía para facilitar la enseñanza explícita de la naturaleza de la ciencia y su integración en el desarrollo del currículo de ciencias. Interaç̧̃̃es, 11(34), 312-349.

Vázquez, Á., Manassero, M.A. (2016). Un modelo formativo para mejorar las ideas de los profesores sobre temas de naturaleza de ciencia y tecnología. Profesorado. Revista de currículum y formación del profesorado, 20, 56-75.

Vázquez, A., \& Manassero, M. A. (2017). An Alternative Conceptualization of the Nature of Science for Science and Technology Education. Conexão Ciência, 12, 18-24.

Vázquez-Alonso, Á., Manassero, M. A. y Bennássar, A. (Comp.) (2014). Secuencias de Enseñanz̧a Aprendizaje sobre la Naturaleza de la Ciencia y la Tecnología. Unidades Didácticas del proyecto EANCYT. Palma de Mallorca: Autor (CD).

Vázquez, Á., Manassero-Mas, M.A., Bennássar-Roig, A. J. (2015). La enseñanza y el aprendizaje de la naturaleza de la ciencia y tecnología (EANCYT): una investigación experimental con perspectiva latina. Interaç̧ões, 11(34), 8-34.

Vesterinen V-M., Manassero-Mas M-A., Vázquez-Alonso Á. (2014). History, Philosophy, and Sociology of Science and Science-Technology-Society Traditions in Science Education: Continuities and Discontinuities. En M. R. Matthews (ed.), International Handbook of Research in History, Philosophy and Science Teaching (pp. 1895-1925). Dordrecht: Springer. 


\section{Anexo}

Análisis comparativo de correspondencia entre las cuestiones representativas de la naturaleza de ciencia y tecnología en la taxonomía 4-mundos y en tres propuestas de la literatura (las correspondencias se han asignado siguiendo la "prueba de mejor ajuste" pues algunos epígrafes se superponen entre varias categorías).

\begin{tabular}{|c|c|c|c|}
\hline Taxonomía 4-Mundos * & $\begin{array}{l}\text { Taxonomía de } \text { RFN ** } \\
\text { (Erduran y Dagher } \\
\text { 2014) }\end{array}$ & Matthews (2012) & McComas (2008) \\
\hline \multicolumn{4}{|l|}{ I. DEFINICIONES } \\
\hline \multicolumn{4}{|l|}{ 1. Ciencia y tecnología } \\
\hline \multicolumn{4}{|l|}{ 01. Ciencia } \\
\hline 02. Tecnología & Tecnología & Tecnología & \\
\hline \multicolumn{4}{|l|}{$03 . \mathrm{I}+\mathrm{D}$} \\
\hline 04. Interdependencia & & & $\begin{array}{l}\text { Cy'T interactúan, } \\
\text { pero son diferen- } \\
\text { tes }\end{array}$ \\
\hline \multicolumn{4}{|l|}{$\begin{array}{l}\text { II. SOCIOLOGÍA EXTER- } \\
\text { NA DE CyT }\end{array}$} \\
\hline \multicolumn{4}{|l|}{ 2. Influencias de la sociedad en CyT } \\
\hline \multicolumn{4}{|l|}{ 01. Gobierno } \\
\hline \multicolumn{4}{|l|}{ 02. Industria } \\
\hline \multicolumn{4}{|l|}{ 03. Ejército } \\
\hline 04. Ética & & Valores & \\
\hline 05. Instituciones educativas & $\begin{array}{l}\text { Respetar las necesidades } \\
\text { humanas }\end{array}$ & & \\
\hline \multicolumn{4}{|l|}{ 06. Grupos de especial interés } \\
\hline $\begin{array}{l}\text { 07. Influencias sobre los } \\
\text { científicos }\end{array}$ & Personal, cultural y social & $\begin{array}{l}\text { Cosmovisiones } \quad y \\
\text { religión }\end{array}$ & \\
\hline 08. Influencias generales & & & $\begin{array}{l}\text { Influencias } \\
\text { históricas, } \\
\text { culturales, sociales }\end{array}$ \\
\hline \multicolumn{4}{|l|}{ 3. Influencia ternaria } \\
\hline 01. Interacción CTS & & $\begin{array}{l}\text { Cuestiones socio- } \\
\text { científicas }\end{array}$ & \\
\hline $\begin{array}{l}\text { 4. Influencias de la ciencia y la tecno- } \\
\text { logía en la sociedad }\end{array}$ & $\begin{array}{l}\text { 4. Influencias de la ciencia } \\
\text { y la tecnología en la socie- } \\
\text { dad }\end{array}$ & & \\
\hline 01. Responsabilidad social & Ventajas y riesgos & & \\
\hline \multicolumn{4}{|l|}{ 02. Decisiones sociales } \\
\hline 03. Problemas sociales & $\begin{array}{l}\text { Satisfacción de las necesi- } \\
\text { dades humanas }\end{array}$ & & \\
\hline \multicolumn{4}{|l|}{ 04. Resolución de problemas } \\
\hline \multicolumn{4}{|l|}{ 05. Bienestar económico } \\
\hline \multicolumn{4}{|l|}{ 06. Contribución al ejército } \\
\hline $\begin{array}{lll}07 . & \text { Contribución } & \text { al } \\
\text { pensamiento social } & \end{array}$ & $\begin{array}{l}\text { Prevenir el control de } \\
\text { ideas por grupos }\end{array}$ & & \\
\hline \multicolumn{4}{|l|}{ 08. Influencias generales } \\
\hline \multicolumn{4}{|l|}{$\begin{array}{l}\text { 5. Influencias de la ciencia escolar en } \\
\text { la sociedad }\end{array}$} \\
\hline 01. Unión dos culturas & $\begin{array}{lcc}\text { Ser } \quad y & \text { actuar } \\
\text { honorablemente } & \\
\end{array}$ & & \\
\hline 02. Empoderamiento social & $\begin{array}{l}\text { Respetar las ideas basadas } \\
\text { en la evidencia } \\
\text { Justificar conclusiones (ra- } \\
\text { zones y datos) }\end{array}$ & & \\
\hline $\begin{array}{l}\text { 03. Caracterización de la ciencia } \\
\text { escolar }\end{array}$ & $\begin{array}{l}\text { Explicaciones precisas } \\
\text { Reconocer objeciones y } \\
\text { practicar autocrítica }\end{array}$ & & \\
\hline
\end{tabular}




\begin{tabular}{|c|c|c|c|}
\hline \multicolumn{4}{|l|}{$\begin{array}{l}\text { III. SOCIOLOGÍA INTER- } \\
\text { NA DE CyT }\end{array}$} \\
\hline \multicolumn{4}{|l|}{ 6. Características de los cientificos } \\
\hline 01. Motivaciones & Aceptación de desafíos & & \\
\hline 02. Valores y estándares & $\begin{array}{l}\text { Objetividad } \\
\text { Fiel y veraz }\end{array}$ & & \\
\hline 03. Creencias & Compromisos teóricos & & \\
\hline 04. Capacidades & $\begin{array}{l}\text { Desarrollo de modelos, } \\
\text { explicaciones, argumenta- } \\
\text { ciones, evaluaciones y re- } \\
\text { flexiones }\end{array}$ & & \\
\hline 05. Efectos de género & & Feminismo & \\
\hline \multicolumn{4}{|l|}{$\begin{array}{l}\text { 06. La infrarrepresentación de } \\
\text { las mujeres }\end{array}$} \\
\hline \multicolumn{4}{|l|}{$\begin{array}{l}\text { 7. Construcción social del conoci- } \\
\text { miento científico }\end{array}$} \\
\hline 01. Colectivización & $\begin{array}{l}\text { Red de instituciones } \\
\text { Examen crítico }\end{array}$ & & \\
\hline 02. Decisiones científicas & $\begin{array}{l}\text { Teoría elección / raciona- } \\
\text { lidad } \\
\text { Anomalías / refutaciones }\end{array}$ & $\begin{array}{l}\text { Elección de la teoría } \\
\text { y racionalidad }\end{array}$ & \\
\hline 03. Comunicación profesional & $\begin{array}{l}\text { Publicación } \\
\text { Evaluación por pares }\end{array}$ & & \\
\hline 04. Competencia profesional & $\begin{array}{l}\text { Esfuerzo humano } \\
\text { Creatividad }\end{array}$ & Creatividad & $\begin{array}{l}\text { La ciencia tiene un } \\
\text { componente crea- } \\
\text { tivo }\end{array}$ \\
\hline 05. Interacciones sociales & $\begin{array}{l}\text { Los científicos son parte } \\
\text { de la sociedad }\end{array}$ & & \\
\hline $\begin{array}{l}\text { 06. Influencias de los } \\
\text { individuos }\end{array}$ & $\begin{array}{l}\text { Campo personal y de dis- } \\
\text { ciplina }\end{array}$ & & \\
\hline 07. Influencias nacionales & $\begin{array}{ll}\begin{array}{l}\text { Diferentes } \\
\text { contribuyen }\end{array} & \text { personas } \\
\end{array}$ & & \\
\hline \multicolumn{4}{|l|}{ 08. Ciencia pública y privada } \\
\hline \multicolumn{4}{|l|}{$\begin{array}{l}\text { 8. Construcción social de la tecnolo- } \\
\text { gía }\end{array}$} \\
\hline \multicolumn{4}{|l|}{ 01. Decisiones tecnológicas } \\
\hline \multicolumn{4}{|l|}{ 02. Autonomía de la tecnología } \\
\hline \multicolumn{4}{|l|}{ IV.EPISTEMOLOGÍA } \\
\hline \multicolumn{4}{|l|}{$\begin{array}{l}\text { 9. Naturaleza del conocimiento } \\
\text { cientifico }\end{array}$} \\
\hline 01. Observaciones & Observación & Base empírica & $\begin{array}{l}\text { Evidencia } \\
\text { empírica }\end{array}$ \\
\hline 02. Modelos científicos & Modelos & Modelos & \\
\hline 03. Esquemas de clasificación & Clasificación & & \\
\hline 04. Tentativa & Cambio & Tentativa & $\begin{array}{l}\text { Provisional, } \\
\text { duradero y auto- } \\
\text { corrector }\end{array}$ \\
\hline 05. Hipótesis, teorías y leyes & Teorías y leyes científicas & $\begin{array}{ll}\begin{array}{l}\text { Teorías y leyes } \\
\text { científicas }\end{array} & \\
\end{array}$ & $\begin{array}{l}\text { Conocimiento } \\
\text { distinto / especial }\end{array}$ \\
\hline 06. Enfoque a la investigación & $\begin{array}{l}\text { Experimentación } \\
\text { Ajuste empírico } \\
\text { Amplio conjunto de } \\
\text { métodos }\end{array}$ & $\begin{array}{l}\text { Método científico } \\
\text { Experimentación }\end{array}$ & $\begin{array}{l}\text { Los experimentos } \\
\text { no son la única } \\
\text { ruta hacia el cono- } \\
\text { cimiento }\end{array}$ \\
\hline 07. Precisión e incertidumbre & $\begin{array}{l}\text { Idealización } \\
\text { Precisión }\end{array}$ & Idealización & \\
\hline 08. Razonamiento lógico & $\begin{array}{l}\text { Elección de teorías y ra- } \\
\text { cionalidad }\end{array}$ & $\begin{array}{l}\text { Elección de teorías y } \\
\text { racionalidad } \\
\text { Explicación }\end{array}$ & $\begin{array}{l}\text { Pruebas inducti- } \\
\text { vas, racionales e } \\
\text { hipotético-deducti- } \\
\text { vas }\end{array}$ \\
\hline
\end{tabular}




\begin{tabular}{|l|l|l|l|}
\hline 09. Suposiciones de la ciencia & $\begin{array}{l}\text { Novedad } \\
\text { Matematización }\end{array}$ & $\begin{array}{l}\text { Dependencia de la } \\
\text { teoría } \\
\text { Matematización }\end{array}$ & $\begin{array}{l}\text { Ningún método } \\
\text { científico } \\
\text { Elemento subjeti- } \\
\text { vo (carga de la teo- } \\
\text { ría) }\end{array}$ \\
\hline 10. Estatus epistemológico & $\begin{array}{l}\text { Cuestiones del mundo na- } \\
\text { tural basadas en eviden- } \\
\text { cias empíricas }\end{array}$ & $\begin{array}{l}\text { La ciencia no pue- } \\
\text { de responder todo }\end{array}$ \\
\hline $\begin{array}{l}\text { 11. Paradigmas y coherencia de } \\
\text { conceptos }\end{array}$ & $\begin{array}{l}\text { Realismo } \\
\text { constructivismo }\end{array}$ & $\begin{array}{l}\text { Ciencia normal y } \\
\text { revoluciones }\end{array}$ \\
\hline
\end{tabular}

* Elaboración por los autores del marco de referencia VOSTS (Aikenhead y Ryan, 1992).

** Algunos nombres se han abreviado para una mejor presentación y por las limitaciones de espacio. 